\title{
Molecular dynamics simulation of discontinuous volume phase transitions in highly-charged crosslinked polyelectrolyte networks with explicit counterions in good solvent
}

\author{
De-Wei Yin, Qiliang Yan, a) and Juan J. de Pablob) \\ Department of Chemical and Biological Engineering, University of Wisconsin-Madison, \\ Madison, Wisconsin 53706-1691
}

(Received 14 June 2005; accepted 2 September 2005; published online 3 November 2005)

\begin{abstract}
The volumetric properties of highly-charged defect-free polyelectrolyte networks with tetrafunctional crosslinks are studied through molecular dynamics simulations in the canonical ensemble. The network backbone monomers, which are monovalent, and the counterions, which are mono-, di-, or trivalent, are modeled explicitly in the simulations, but the solvent is treated implicitly as a dielectric medium of good solvation quality. The osmotic pressure of the network-solvent system is found to depend greatly on the strength of electrostatic interactions. Discontinuous volume phase transitions are observed when the electrostatic interactions are strong, and the onset of these transitions shifts to higher solvent dielectricity as the counterion valency increases. The roles of the various virial contributions to the osmotic pressure are examined. The network elasticity entropy is found to behave nearly classically. As the network contracts and collapses with increasing strength of electrostatic interactions, the loss of counterion entropy leads to increased counterion osmotic pressure contributions via two mechanisms. The reduction in available configurational space increases the counterion translational entropy contribution to the ideal part of the osmotic pressure, and the greater number of counterion-monomer contacts formed due to counterion condensation and confinement increases the counterion excluded-volume entropy contribution to the excess part of the osmotic pressure. These observations contrast the decrease in the single ideal-gas-like counterion translational entropy contribution to the osmotic pressure predicted by the counterion condensationcharge renormalization theory. An accompanying decrease in the total electrostatic energy balances the loss of counterion excluded-volume entropy as the polyelectrolyte networks collapse in low-dielectric solvents. This interplay between the electrostatic energy and the counterion excluded-volume entropy appears to be responsible for the discontinuous volume phase transitions that are observed in polyelectrolyte networks. The structure of the polyelectrolyte network is also found to be affine in the swollen state, with constituent chains nearly fully extended, and nonaffine in the collapsed state, with the chains adopting a Gaussian conformation.
\end{abstract}

(C) 2005 American Institute of Physics. [DOI: 10.1063/1.2102827]

\section{INTRODUCTION}

A crosslinked polyelectrolyte network consists of a chemically and permanently crosslinked backbone of polyelectrolyte chains with ionizable functional groups. Counterions that dissociate from these ionizable groups are entrapped within the network and ensure its overall electrical neutrality. The solvent that fills the interstitial space of the network may also contain other dissolved salts. Crosslinked polyelectrolyte networks that are swollen with an absorbed solvent are referred to as crosslinked polyelectrolyte gels.

Natural polyelectrolyte gels are found in living organisms where they have biochemical functions such as the regulation of the transport and storage of water and electrolytes, or mechanical functions such as lubrication and shock

\footnotetext{
${ }^{a)}$ Present address: Synopsys, Inc., 2025 NW Cornelius Pass Rd., Hillsboro, OR 97124

b) Author to whom correspondence should be addressed. Electronic mail: depablo@engr.wisc.edu
}

protection. ${ }^{1}$ Synthetic polyelectrolyte gels have been manufactured industrially for many applications. The most wellknown use of synthetic polyelectrolyte gels is in superabsorbent materials for hygienic and medical products, which capitalize on the ability of the gel to absorb a large amount of water and swell hundredfold in volume. ${ }^{2-5}$ Other applications include uses as surfactants and flocculants in water treatment, as microcapsules for drugs or fertilizers, as enzyme immobilization matrices in bioreactors, as agents used in the separation and purifcation of proteins, and as electrolyte media in batteries. ${ }^{2-5}$

While the swelling behavior of weakly-charged polyelectrolyte networks manifests as a continuous volume transition, as it is in neutral polymer networks or gels, the volume transition of highly-charged polyelectrolyte networks is known to be discontinuous and of first order, the onset of which depends on factors including temperature, pressure, solvent composition, exposure to light or to electric fields, ${ }^{1,6}$ and application of tensile forces. ${ }^{7-10}$ A series of reports on the experimental observation of the discontinuous volume 
phase transition in polyacrylamide and polyacrylic acid networks was first presented by Tanaka et al. ${ }^{1,6,11-13}$ A general theoretical prediction of the volume phase transition phenomenon in polymer networks formed by crosslinking linear polymer chains that are swollen in a solvent had been presented by Dušek and Patterson, ${ }^{14}$ and several theoretical models concerning phase transitions in polyelectrolyte gels had been presented earlier by Hill in the context of muscle contraction and the $\alpha-\beta$ transformation in fibrous proteins. ${ }^{7-9}$

Theories on neutral polymer networks are relatively well established $^{15-18}$ and have been verified by computer simulation studies. ${ }^{19-24}$ The development of theories on linear polyelectrolyte chains and on polyelectrolyte networks, however, has emerged more slowly. The principal difficulty in dealing with polyelectrolytes lies in the treatment of long-range electrostatic interactions, which has necessitated the use of approximate models. ${ }^{25-33}$ During the last 15 years, computer simulations of linear polyelectrolyte chains have allowed researchers to assess the validity of these approximations. ${ }^{34-40}$

For polyelectrolyte networks, much of past theoretical work has focused on the swelling behavior of polyelectrolyte gels. The network elasticity, polymer-solvent mixing, and counterion translational entropy contributions to the free energy are generally assumed to be separable and additive..$^{9,13,15,41-43}$ Treatments of the swelling behavior of polyelectrolyte gels often idealize the polymer-polymer and polymer-solvent interactions and neglect the electrostatic interactions within and between constituent polyelectrolyte chains, leading to the assumption that the chain conformations are Gaussian and that the positions of crosslink nodes follow the extension of the entire network according to the principle of affine deformation. ${ }^{12,13,15}$ To improve upon these assumptions, Hooper and co-workers introduced additional types of monomer and solvent contact sites into the conventional lattice-mixing theory to account for the effects of hydrogen bonding in hydrophilic polymer solutions and hydrogels. ${ }^{44,45}$ Marchetti et al. also included empty lattice sites in the lattice-mixing model to allow for the volume compressibility required to predict the lower consolute volume phase transition observed in certain temperaturesensitive nonionic hydrogels. ${ }^{46,47}$ Otherwise, the electrostatic effects between charged sites in polyelectrolyte gels have generally been considered insofar as they impose an electroneutrality constraint that confines counterions within the oppositely charged backbone network; this condition is analogous to that of a Donnan membrane equilibrium and gives rise to an ideal-gas-like counterion translational entropy term. ${ }^{11,13,15,48}$

Network elasticity and counterion translational entropy give rise to smooth volume transitions in polyelectrolyte networks and show that polyelectrolyte gels have greater swelling capacity than their neutral counterparts. Discontinuous volume phase transitions have been predicted in polyelectrolyte networks when poor polymer-solvent mixing interactions are also included in the analysis (without considering electrostatics beyond the Donnan equilibria analogy stated previously), ${ }^{11}$ but the reason for the transition in such networks should be attributed to the tendency for backbone monomers to aggregate, the same reason for which the transitions are predicted and observed in neutral polymer networks. $^{22,49}$

Only a few numerical simulations of polyelectrolyte networks and gels have so far been reported in literature. The Monte Carlo simulation by Aalberts employed the bondfluctuation model to represent crosslinked chains on a twodimensional lattice without explicitly considering counterions. $^{50}$ Our previous work using three-dimensional lattice-based Monte Carlo simulations of large polyelectrolyte networks in the presence of explicit counterions showed the first numerical evidence of discontinuous volume phase transitions in polyelectrolyte networks. ${ }^{51}$ Schneider and coworkers presented continuum-space Monte Carlo simulations of smaller samples of polyelectrolyte gels ${ }^{52-55}$ in which they investigated the swelling behavior of polyelectrolyte gels with respect to various parameters. Their more recent results showed discontinuous volume phase transitions in polyelectrolyte networks consisting of crosslinked 20-mer polyelectrolyte chains, particularly in cases where the dielectric constant of the implicit solvent was small and a short-range attraction between backbone monomers was provided using a square-well potential. Mann et al. recently presented scaling relations for the equilibrium swelling of polyelectrolyte gels of various chain lengths and charge densities with monovalent counterions using data generated from canonical and isothermal-iso-osmotic molecular dynamics simulations. ${ }^{56,57}$ In developing these scaling relations, they calculated an effective counterion osmotic pressure based on the renormalization of charges due to counterion condensation, but their work did not address the issue of volume phase transitions. The first report on simulations of polyelectrolyte networks with explicit counterions and explicit solvent particles is that by $\mathrm{Lu}$ and Hentschke. They used a two-box-particle transfer molecular dynamics method to simulate an extremely highly crosslinked network of monomeric chains in equilibrium with a dipolar Stockmayer solvent. $^{58}$

In this work we use molecular dynamics simulations in the canonical ensemble to simulate highly-charged polyelectrolyte networks with explicit mono-, di-, or trivalent counterions in an implicit solvent of good solvation quality and adjustable dielectricity. Using the data generated by these simulations, we gain some insight into the driving forces that appear to be responsible for the discontinuous volume phase transition observed in polyelectrolyte networks and reveal some structural aspects of polyelectrolyte networks in the swollen and collapsed states. Based on these observations and analysis, we comment on the validity of the assumptions implicit in various theoretical treatments.

\section{MODEL AND SIMULATION METHOD}

The model considered in this work consists of a defectfree crosslinked network of polyelectrolyte chains forming a diamond-lattice tetrahedral topology, the same as the topology used in several previous numerical studies. ${ }^{51-57}$ Eight tetrafunctional nodes and 16 linear polyelectrolyte chains of 98 or 99 monomers (depending on the valency of the 
counterions) crosslinked at the ends make up one topologically repeatable unit cell of the network for the purpose of applying the periodic boundary condition. Each monomer on the crosslinked backbone is anionic and monovalent, and an appropriate number of oppositely charged mono-, di-, or trivalent counterions $\left(q_{\mathrm{i}}=1,2,3\right)$ ensures the electroneutrality of the system. Otherwise, our model resembles the restricted primitive model of electrolytes ${ }^{9,59}$ insofar as all particles have the same diameter $\sigma$ and the same mass $m$, and the solvent is treated as an implicit dielectric continuum with dielectric constant $\epsilon$. The particles, however, are modeled as soft spheres rather than hard spheres, as explained below. Because of the computational demands of calculating the electrostatic interactions, we performed most simulations on systems consisting of a single repeatable unit cell; several simulations of larger cubic assemblages of eight unit cells, run for shorter simulation times, gave results that were consistent with those of the single-unit-cell systems. In all cases periodic boundary conditions were applied to simulate a network of infinite size.

The total potential energy of the system includes three contributions. The short-range interaction between any two particles separated by a distance $r$ is modeled by the WeeksChandler-Andersen (WCA) potential ${ }^{60-62}$

$$
u_{\mathrm{WCA}}(r)= \begin{cases}4 \varepsilon\left[\left(\frac{\sigma}{r}\right)^{12}-\left(\frac{\sigma}{r}\right)^{6}+\frac{1}{4}\right], & r<\sqrt[6]{2} \sigma ; \\ 0, & r \geqslant \sqrt[6]{2} \sigma .\end{cases}
$$

The purely repulsive nature of this interaction implies that the particles are immersed in a good solvent. ${ }^{36,37}$ The polyelectrolyte chains are modeled as freely jointed chains whose monomers are connected by finitely extendible nonlinear elastic (FENE) springs. The energy of a FENE bond is given by $^{63,64}$

$$
u_{\text {bond }}(r)=-\frac{1}{2} k_{\text {bond }} r_{\text {bond }}^{2} \ln \left[1-\left(\frac{r}{r_{\text {bond }}}\right)^{2}\right] .
$$

In our simulations, the FENE spring constant is $k_{\text {bond }}$ $=10 k_{\mathrm{B}} T / \sigma^{2}$; the maximum bond length is $r_{\text {bond }}=1.5 \sigma$, thereby ensuring that the constituent chains cannot cross through one another. The electrostatic energy between two electrically charged particles of valencies $q_{1}$ and $q_{2}$ and separated by a distance $r$ is

$$
u_{\mathrm{elec}}(r)=\frac{e^{2}}{4 \pi \epsilon} \frac{q_{1} q_{2}}{r}=\lambda k_{\mathrm{B}} T \frac{q_{1} q_{2}}{r},
$$

where $\lambda=e^{2} / 4 \pi \epsilon k_{\mathrm{B}} T$ is the Bjerrum length, $e$ is the elementary charge, and $\epsilon$ is the effective dielectric constant of the solvent. The Bjerrum length, at which distance two unit charges have an interaction energy $\pm k_{\mathrm{B}} T$, provides a measure of the strength of electrostatic energy relative to the thermal energy of the system. The electrostatic interactions are evaluated using an optimized Ewald summation method, ${ }^{65-68}$ typically with a real-space cutoff of $0.3 V^{1 / 3}=0.3 \mathrm{~L}$, a reciprocalspace cutoff of 10, and an Ewald convergence parameter of $10 \mathrm{~V}^{-1 / 3}=10 / \mathrm{L}$ to ensure a relative error of less than $1 \times 10^{-4}$ in the electrostatic energy.
The molecular dynamics simulations are performed in the canonical ensemble where the integration of the equations of motion is carried out using the Nosé-Hoover chains algorithm. ${ }^{67,69,70}$ The size of the time steps used depends on the stiffness of the system and falls between $\tau / 1024$ and $\tau / 128$, where $\tau=\sigma \sqrt{m / \varepsilon}$. The Nosé-Hoover thermostat is set at $T=\varepsilon / k_{\mathrm{B}}$. Most of the data points are obtained from multiple simulations each lasting at least $8 \times 10^{6}$ time steps. The initial configuration is constructed at a low density by placing the crosslink nodes onto a diamond lattice and then connecting them with straight polyelectrolyte chains. The system is then gradually compressed to the desired density and allowed to equilibrate before sample statistics are collected.

\section{RESULTS AND DISCUSSION}

Figure 1 shows the osmotic pressure of polyelectrolyte networks with mono-, di-, and trivalent counterions as a function of the network density and the Bjerrum length, at a temperature of $k_{\mathrm{B}} T / \varepsilon=1$. The osmotic pressure $\pi$ is calculated as

$$
\begin{aligned}
\pi= & \left(\rho_{\mathrm{nw}}+\rho_{\mathrm{i}}\right) k_{\mathrm{B}} T+\frac{1}{3 V}\left(\sum_{\text {bond }}\langle\boldsymbol{r} \cdot \boldsymbol{F}\rangle_{\text {bond }}\right. \\
& \left.+\sum_{\mathrm{WCA}}\langle\boldsymbol{r} \cdot \boldsymbol{F}\rangle_{\mathrm{WCA}}+\left\langle U_{\text {elec }}\right\rangle\right) .
\end{aligned}
$$

In Equation (1), $\rho_{\mathrm{nw}}$ and $\rho_{\mathrm{i}}$ are the number densities of the network backbone monomers and of the counterions. The two $\langle\boldsymbol{r} \cdot \boldsymbol{F}\rangle$ terms and $\left\langle U_{\text {elec }}\right\rangle$ are the virial terms due to FENE bonds, the WCA dispersion, and the Coulombic interactions. For a polyelectrolyte network to be in thermodynamic equilibrium with an implicit solvent bath, the free energy $F$ of the entire network-counterion-solvent system must be at a minimum; thus

$$
\pi=-\left(\frac{\partial F}{\partial V}\right)_{T, \lambda}=0,
$$

and the osmotic pressure $\pi$ must be identically zero. If $\pi>0$, the network swells by absorbing solvent until the osmotic pressure reduces to zero. If $\pi<0$, then the system is unstable and contracts by expelling pure solvent until the osmotic pressure rises to zero. In the low-density limit, the isotherms approach a large negative osmotic pressure and elastic forces dominate the behavior of the forcibly expanded network. At high densities the osmotic pressure tends to a large positive value due to excluded-volume repulsions between the particles. Simulation of polyelectrolyte networks near these extreme limits is difficult inasmuch as the system becomes stiff in the former case and is possibly confined to a local part of phase space in the latter. The volume transitions of interest occur in more moderate ranges of density, and it is on these conditions that we focus our discussion.

\section{A. Osmotic pressure and volumetric behavior}

The osmotic pressure-density relationship shown in Fig. 1 exhibits a strong dependency on the strength of electrostatic interactions and on the valency of the counterions. At short Bjerrum lengths $\lambda$, which implies a solvent with a 

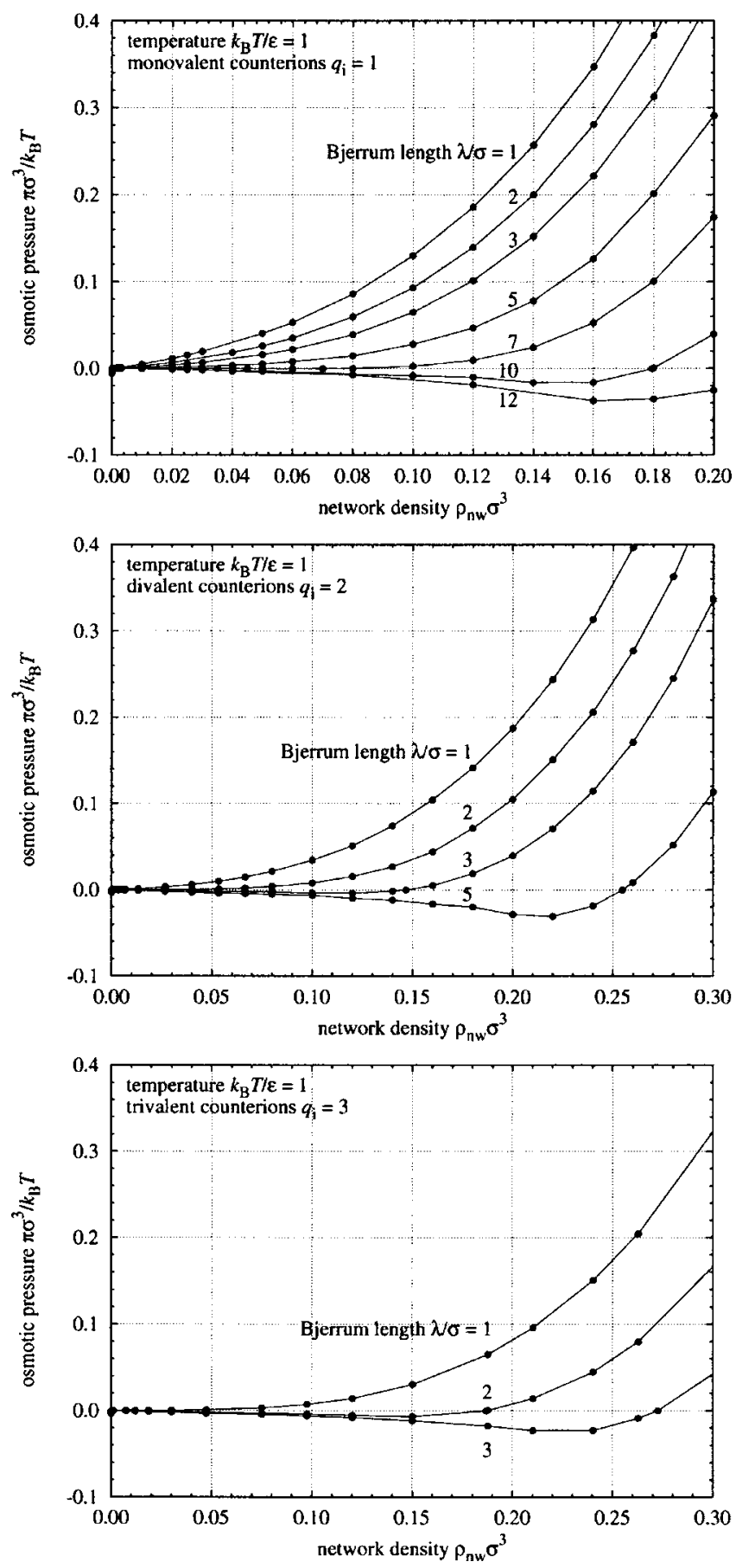

FIG. 1. Osmotic pressure $\pi$ vs network density $\rho_{\mathrm{nw}}$ polyelectrolyte networks at various Bjerrum lengths $\lambda$ at a temperature of $T=\varepsilon / k_{\mathrm{B}}$. The errors are smaller than the size of the symbols.

large dielectric constant $\epsilon$, the osmotic pressure along an isotherm increases monotonically as a function of density. The isotherm intersects $\pi=0$ at a low density, indicating that the network is swollen at equilibrium. As the strength of the electrostatic interactions increases due to an increase in either the Bjerrum length or in the valency of the counterions, the isotherms shift to lower osmotic pressures and the equilibrium state point of the swollen gel moves along the $\pi=0$ line to higher densities. For a given Bjerrum length, the lowering of the osmotic pressure is more pronounced for the higher counterion valency than it is for the lower valency. At

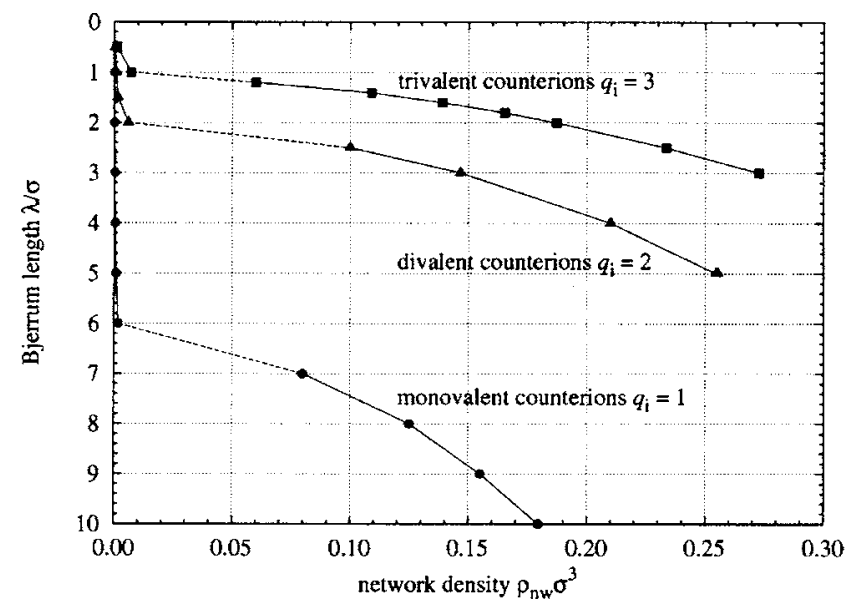

FIG. 2. Equilibrium states of polyelectrolyte networks at $T=\varepsilon / k_{\mathrm{B}}$ and $\pi=0$. The dotted line segments indicate the approximate regions along the curves where the discontinuous phase transitions occur; the actual phase transitions would occure across horizontal tie-lines.

long Bjerrum lengths the isotherms intersect $\pi=0$ at relatively high densities where the networks assume a collapsed state at equilibrium.

We should point out that the purpose of our deliberate adjustment of the Bjerrum length in these simulations, without making any change to the repulsive WCA dispersion interaction between particles, is to isolate the effect of the strength of electrostatic interactions on the overall behavior of hydrophilic polyelectrolyte networks. Variations in the Bjerrum length alone cannot capture entirely the effects of changes in the solvent quality or the addition of salt. A change in the solvent quality in actual physical experiments, such as the dilution of water using acetone in Tanaka's early experiments, ${ }^{11,12}$ would necessarily entail changes in both the solvent dielectricity and the interparticle dispersion interactions; in such cases both the Bjerrum length and the hydrophobicity of the network backbone would increase with increasing acetone concentration in the solvent. Simulations in the context of added salts, which may also involve modifications in the Bjerrum length and the dispersion interactions, will also require independent simulations of the salt solutions without the presence of the polyelectrolyte network, so that the chemical potentials of the salt species can be equated inside and outside the polyelectrolyte network in order to establish thermodynamic equilibria. ${ }^{55}$

The equilibrium state points on the $\pi=0$ abscissae in Fig. 1 are extracted and plotted in Fig. 2 to show more clearly the dependency of the equilibrium volumetric behavior of the networks on the Bjerrum length and on the counterion valency.

\section{B. Van der Waals instability and volume phase transition}

When the strength of the electrostatic interactions is sufficiently strong, an unstable region where $(\partial \pi / \partial \rho)_{T, \lambda}<0$ develops along the isotherms. This is illustrated in the isotherms for $\lambda / \sigma=7,10$, and 12 when $q_{\mathrm{i}}=1$, for $\lambda / \sigma=3$ and 5 when $q_{\mathrm{i}}=2$, and for $\lambda / \sigma=2$ and 3 when $q_{\mathrm{i}}=3$ in Fig. 1. 
In theory a Maxwell equal-area construction can be performed in this van der Waals loop, from which two state points of equal free energy can be found at the two ends of the tie-line. Only for a particular Bjerrum length $\lambda_{\text {trans }}$ does the constructed Maxwell tie-line coincide exactly with $\pi=0$; in this special case the two end points correspond to the actual equilibrium states at the two ends of the discontinuous volume phase transition of the network. In practice the value of $\lambda_{\text {trans }}$ is unknown and cannot be isolated using numerical simulation techniques. Unlike the case for vapor-liquid equilibria, the swollen and collapsed equilibrium states cannot coexist in a real macroscopic network because of the elastic resistance against phase nucleation. ${ }^{42}$ The polyelectrolyte network will either swell or collapse completely, although the kinetics for such transitions is known to be very slow. ${ }^{12}$

For any other isotherm of Bjerrum lengths $\lambda \neq \lambda_{\text {trans }}$, whose van der Waals loop crosses the $\pi=0$ line multiple times, both stable equilibrium and metastable states may exist. ${ }^{7,12,54}$ Schneider and Linse were able to locate several of these metastable states using Monte Carlo simulations. ${ }^{54} \mathrm{We}$ believe that the metastable states reported in their work are enhanced by the smaller size of their simulated systems and by the attractive square-well potential interaction that they use for monomer-monomer pairs.

\section{Swelling-collapse mechanism}

In order to gain a better understanding of the mechanism that causes discontinuous volume phase transitions in polyelectrolyte networks, we draw an analogy to vapor-liquid equilibria in simple fluids and examine the interplay between various contributions to the osmotic pressure and to the virial of the system as we vary the Bjerrum length and density along the equilibrium line $\pi=0$. In simple fluids, the coexistence of liquid and vapor in equilibrium is necessarily governed by a balance of energetic and entropic effects in the system: the higher energy in the vapor phase is balanced by a greater entropy while the lesser entropy in the liquid phase is balanced by the lower energy. A similar balance occurs between energy and entropy in the discontinuous volume phase transitions of polyelectrolyte networks.

According to classical theories on rubber elasticity, the swelling of neutral polymer networks is governed by the balance between two effects: the elasticity of the polymer network and the osmotic pressure of an equilvalent polymer solution at the same monomer concentration. Both of these effects are entropic in origin, and their associated entropies depend monotonically on the density: the former tends to contract the network while the latter tends to expand it. ${ }^{15}$ Several theories concerning the swelling of polyelectrolyte gels have built on this framework, replacing the osmotic pressure of the equivalent polymer solution by an osmotic pressure term that corresponds to the translational entropy of the counterions. ${ }^{15}$ By virtue of the typically large concentration of counterions in a polyelectrolyte network system, the network elasticity must yield to a greater expansive force than is found in neutral networks, and a greater degree of swelling is therefore predicted in polyelectrolyte gels. As an extension to this theoretical framework, a decrease in the osmotic pressure contribution associated with the counterion translational entropy as the Bjerrum length increases has been suggested based on counterion condensation and charge renormalization considerations. ${ }^{54,56,57}$ As we shall show later, such an interpretation does not take into consideration the energetic effects of the electrostatic interactions, which play a significant role in discontinuous volume phase transitions in polyelectrolyte networks.

By assuming that the various contributions to the system free energy are additive, which is the inherent assumption made in molecular dynamics or Monte Carlo simulations, the equilibrium condition for the network-solvent system stated in Eq. (2) becomes

$$
\pi=\pi_{\mathrm{id}}+\pi_{\mathrm{ex}}=\pi_{\mathrm{id}}+\left(\pi_{\mathrm{nw}}+\pi_{\mathrm{i}}^{\mathrm{ev}}+\pi_{\text {elec }}\right)=0,
$$

where

$$
\begin{aligned}
& \pi_{\mathrm{id}}=\pi_{\mathrm{mm}}^{\mathrm{id}}+\pi_{\mathrm{i}}^{\mathrm{id}}, \\
& \pi_{\mathrm{nw}}=\pi_{\mathrm{bond}}+\pi_{\mathrm{mm}}^{\mathrm{ev}}, \\
& \pi_{\mathrm{i}}^{\mathrm{ev}}=\pi_{\mathrm{im}}^{\mathrm{ev}}+\pi_{\mathrm{ii}}^{\mathrm{ev}} .
\end{aligned}
$$

In Eqs. (3) and (4) the abbreviations id, ex, nw, ev, elec, m, i, $\mathrm{mm}$, ii, and im refer to ideal, excess, network, excluded volume, electrostatic, monomer, counterion, monomer-monomer pair, counterion-counterion pair, and counterion-monomer pair, respectively. The term $\pi_{\mathrm{id}}=\rho k_{\mathrm{B}} T=\left(\rho_{\mathrm{nw}}+\rho_{\mathrm{i}}\right) k_{\mathrm{B}} T>0$ represents the ideal entropic contribution to the osmotic pressure arising from the intrinsic translational degrees of freedom of the backbone monomers and the counterions. It follows from Eq. (3) that the excess osmotic pressure must be negative under equilibrium conditions, i.e., $\pi_{\mathrm{ex}}=\left(\pi_{\mathrm{nw}}\right.$ $\left.+\pi_{\mathrm{i}}^{\mathrm{ev}}+\pi_{\text {elec }}\right)=-\pi_{\mathrm{id}}=-\rho k_{\mathrm{B}} T<0$, and it must include the energetic effects of the system in order to predict discontinuous volume phase transitions in the network.

In their recent scaling analysis on the swelling in polyelectrolyte networks, Mann et al. ${ }^{57}$ regrouped the various terms in Eqs. (3) and (4) into two groups: a network gel term and a counterion gas term. Such a partition follows naturally from the aforementioned theoretical framework established by Flory. ${ }^{15}$ To examine the interplay between the various excess terms which contribute to the osmotic pressure, we prefer to maintain the three-part $\pi_{\mathrm{nw}}+\pi_{\mathrm{i}}^{\mathrm{ev}}+\pi_{\text {elec }}$ breakdown of the excess term in Eq. (3) and find it useful to normalize Eq. (3) with respect to $\rho k_{\mathrm{B}} T$ to obtain

$$
\frac{\pi}{\rho k_{\mathrm{B}} T}=1+\frac{1}{k_{\mathrm{B}} T}\left(w_{\mathrm{nw}}+w_{\mathrm{i}}^{\mathrm{ev}}+w_{\text {elec }}\right)=0 .
$$

The three quantities

$$
\begin{aligned}
w_{\mathrm{nw}} & =w_{\mathrm{bond}}+w_{\mathrm{mm}}^{\mathrm{ev}} \\
& =-\frac{1}{3 N} \sum_{\text {bond }}\left(\frac{k_{\mathrm{bond}} r^{2}}{1-\left(r / r_{\mathrm{bond}}\right)^{2}}\right)+\frac{8 \varepsilon}{N} \sum_{\mathrm{mm}}^{\dagger}\left(\frac{2 \sigma^{12}}{r^{12}}-\frac{\sigma^{6}}{r^{6}}\right),
\end{aligned}
$$




$$
\begin{aligned}
w_{\mathrm{i}}^{\mathrm{ev}} & =w_{\mathrm{im}}^{\mathrm{ev}}+w_{\mathrm{ii}}^{\mathrm{ev}} \\
& =\frac{8 \varepsilon}{N}\left[\sum_{\mathrm{im}}^{\dagger}\left(\frac{2 \sigma^{12}}{r^{12}}-\frac{\sigma^{6}}{r^{6}}\right)+\sum_{\mathrm{ii}}^{\dagger}\left(\frac{2 \sigma^{12}}{r^{12}}-\frac{\sigma^{6}}{r^{6}}\right)\right], \\
w_{\text {elec }} & =\frac{1}{3 N}\left\langle U_{\text {elec }}\right\rangle,
\end{aligned}
$$

are normalized internal virials ${ }^{66}$ and include the constant factor of $1 / 3$ that appeared earlier in Eq. (1). The daggers next to the operators in Eq. (6) indicate that the summations include only short-range interaction terms for which $r / \sigma<\sqrt[6]{2}$. Whereas the osmotic pressure terms in Eq. (3) span several orders of magnitude in range because of the density scaling, the normalized virial terms in Eqs. (5) and (6) all fall within an order of magnitude and are easier to analyze and interpret.

The values of the three virial terms in Eq. (6), readily available from the results of molecular dynamics simulations, are plotted against the Bjerrum length in Fig. 3 and against the total density $\rho$ in Fig. 4. Both figures show that $w_{\mathrm{i}}^{\mathrm{ev}}$ and $w_{\text {elec }}$ arising from the counterion excluded volume and the electrostatic interactions approximately cancel each other, essentially leaving the network elasticity $w_{\mathrm{nw}} \sim-k_{\mathrm{B}} T$ alone to balance the ideal term in Eq. (5). Furthermore, the magnitudes of both $w_{\mathrm{i}}^{\mathrm{ev}}$ and $w_{\text {elec }}$ are small at short Bjerrum lengths and low densities where the network is swollen, and the corresponding osmotic pressure terms $\pi_{\mathbf{i}}^{\mathrm{ev}}$ and $\pi_{\text {elec }}$ have even smaller magnitudes because of the multiplication by density. Given the small magnitudes of and the cancellation between $w_{\mathrm{i}}^{\mathrm{ev}}$ and $w_{\text {elec }}$, the swelling behavior of weaklycharged polyelectrolyte gels has been explained with apparent success in terms of the balance between an ideal-gas-like counterion osmotic pressure and the network elasticity. ${ }^{5,57}$

The relative invariance in the network virial term $w_{\text {nw }}$ with respect to both the Bjerrum length $\lambda$ and the density $\rho$ in Figs. 3 and 4 is actually consistent with classical rubber theory in that the elastic energy and, by extension, the normalized elastic virial $w_{\text {nw }}$ are independent of the network density $\rho$. The entropic nature of the network contribution enters through the multiplication of $w_{\text {nw }}$ by the total density $\rho$ to give the corresponding osmotic pressure term $\pi_{\mathrm{nw}}=\rho w_{\mathrm{nw}}$. Inasmuch as $w_{\mathrm{nw}} \sim-k_{\mathrm{B}} T$, the resulting osmotic pressure contribution $\pi_{\text {nw }} \sim-\rho k_{\mathrm{B}} T \sim-\pi_{\text {id }}$ reflects the nearly ideal increase in network entropy as the density increases and the constituent chains in the network are allowed to relax in a larger conformational space. The deviation of $w_{\text {nw }}$ from the ideal neutral network value of $-k_{\mathrm{B}} T$ in Figs. 3 and 4 is due to the fact that $w_{\text {nw }}$, which includes only direct contributions from FENE bond interactions $\left(w_{\text {bond }}\right)$ and monomermonomer excluded-volume interactions $\left(w_{\mathrm{mm}}^{\mathrm{ev}}\right)$, as shown in Eq. (6a), is not entirely independent of electrostatic effects. The electrostatic repulsion between adjacent monomers increases the bond length, which decreases $w_{\text {bond }}$ and causes $w_{\text {nw }}$ to be less than $-k_{B} T$; this effect becomes more pronounced as the Bjerrum length increases, resulting in the slightly negative slopes of $w_{\text {nw }}$ for $q_{\mathrm{i}}=1$ and 2 in Fig. 3. In the case of $q_{\mathrm{i}}=3$, however, the electrostatic repulsion between monomers is more effectively screened by the
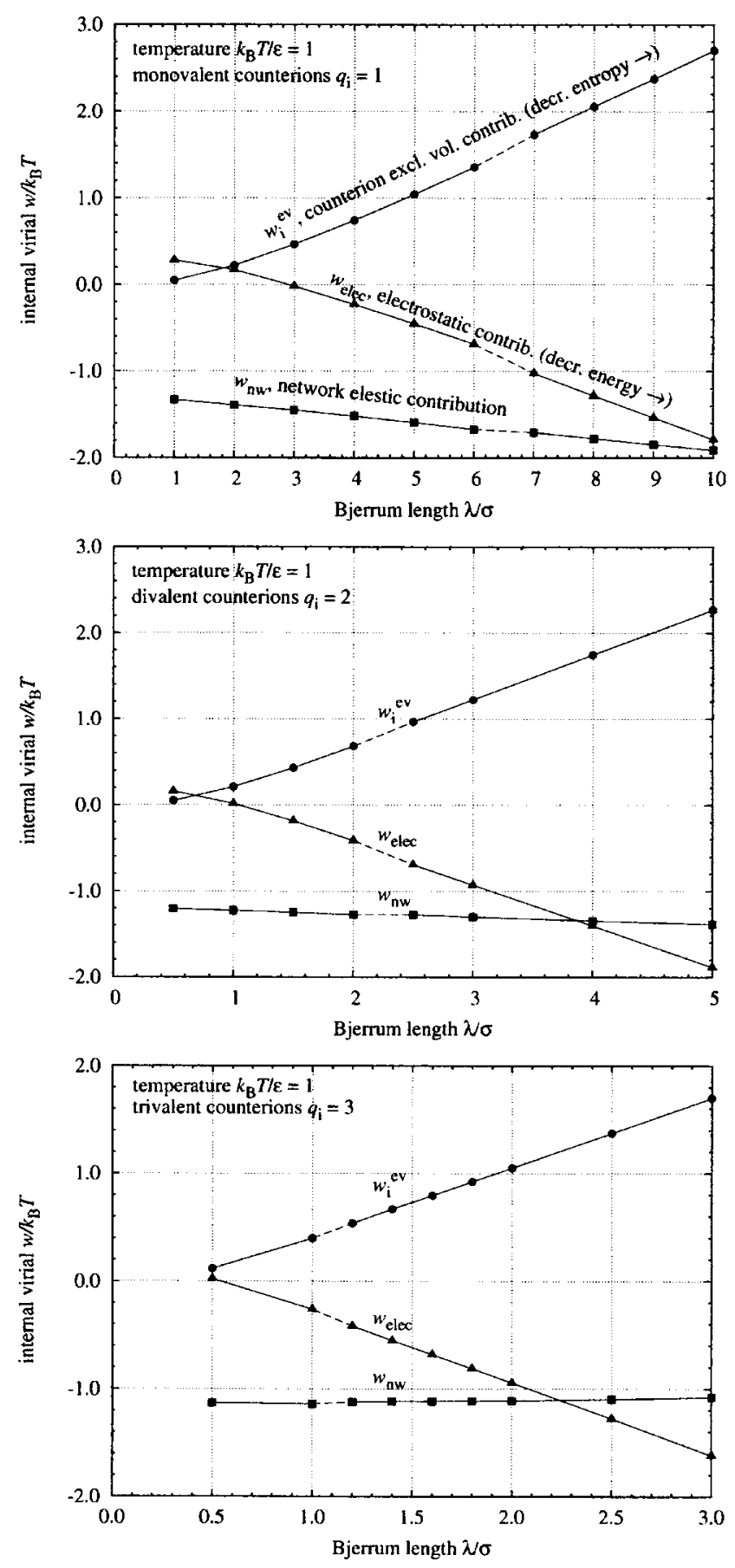

FIG. 3. Variations in the terms of the internal virial $w$ with respect to the Bjerrum length under equilibrium conditions.

trivalent counterions, such that $w_{\text {nw }}$ is essentially constant for all values of the Bjerrum length and the density.

The positive slope of the curves for the counterion excluded-volume virial $w_{\mathrm{i}}^{\mathrm{ev}}$ in Figs. 3 and 4 reflects the loss of entropy due to the condensation and eventual confinement of counterions as the networks are reduced from swollen states to collapsed states. It is important to realize that the overall loss of counterion entropy as the network contracts and collapses consists of two parts: the loss of translational entropy due to the reduction of the available physical volume and hence configurational space of the system, and the loss of excluded-volume entropy due to the lesser mobility of the 

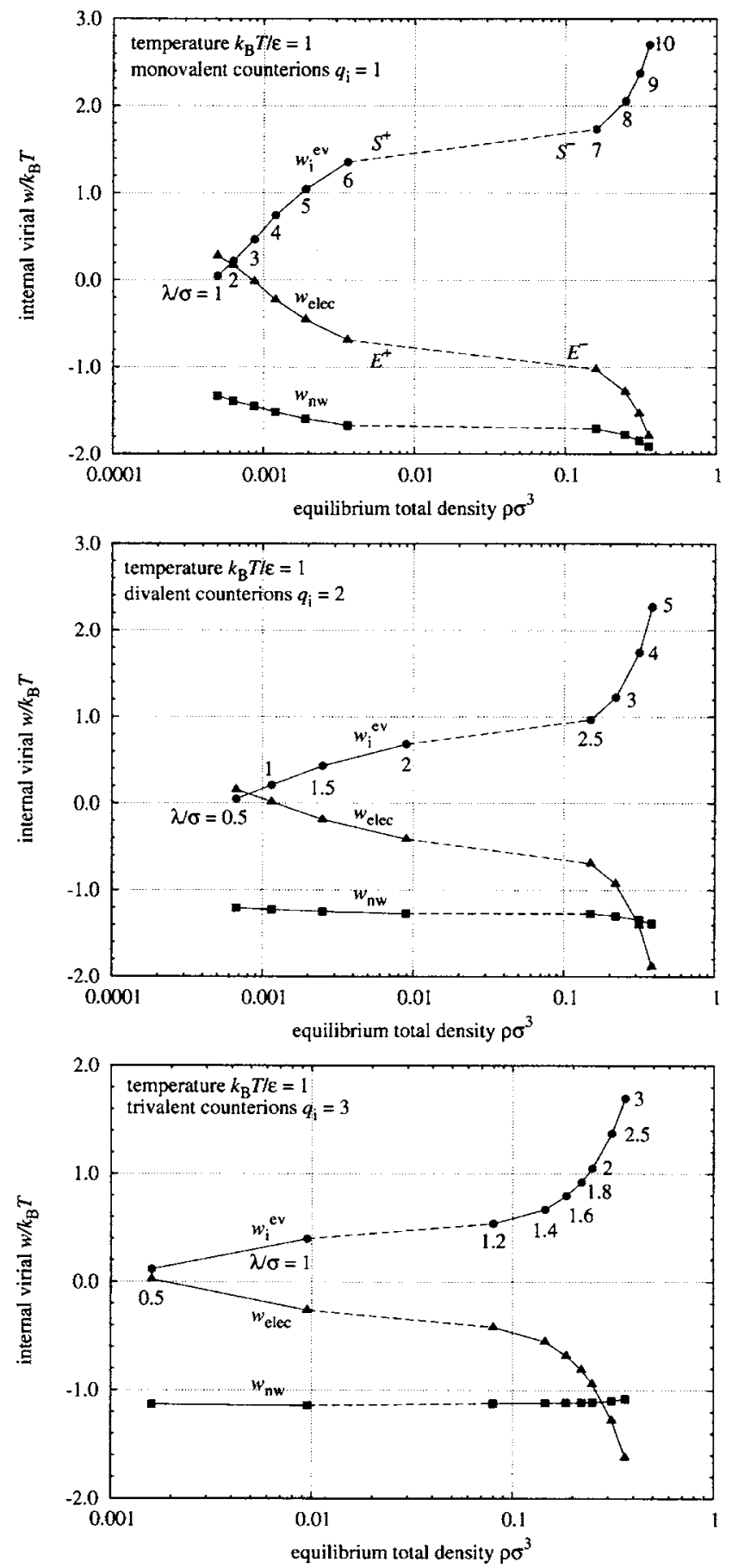

FIG. 4. Variations in the terms of the internal virial $w$ with respect to total density $\rho$ under equilibrium conditions. The numbers next to the symbols along the upper curve are the values of the Bjerrum length $\lambda$ for the three data points at that density.

counterions because of condensation and confinement. Of these two types of counterion entropy losses, the former is associated with an increase in the ideal osmotic pressure contribution $\pi_{\mathrm{i}}^{\mathrm{id}}$ which is proportional to the density $\rho$, while the latter is correlated to an increase in $\pi_{\mathrm{im}}^{\mathrm{ev}}$ that depends on the number of counterion-monomer contacts formed. This dependency on the number of counterion-monomer contacts formed in the latter case is the reason for the positive slope of $w_{\mathrm{i}}^{\mathrm{ev}}$ in Figs. 3 and 4. The condensation of counterions does not therefore decrease the counterion translational entropy contribution to the osmotic pressure, in contrast with the ideal-gas and charge renormalization descriptions of counterions in polyelectrolyte gels recently proposed in literature. ${ }^{54,56,57}$

With both $w_{\text {nw }}$ and $w_{i}^{\text {ev }}$ being attributed to entropic effects, we find that the remaining term $w_{\text {elec }}$ embodies important energetic effects in polyelectrolyte networks. By definition of the internal virial we have $w_{\text {elec }} \propto\left\langle U_{\text {elec }}\right\rangle$, so the osmotic pressure contribution arising from electrostatic interactions is closely associated with the energetics of the system. The electrostatic virial $w_{\text {elec }}$ decreases as the Bjerrum length and density increase because of greater electrostatic cohesion and more effective screening of charges in the system.

While the variation in each of the three virial terms appears to be almost linear with respect to the Bjerrum length in Fig. 3, abrupt changes in the shapes of the curves corresponding to the discontinuous volume phase transitions are revealed clearly in Fig. 4, and the interplay between energy and entropy becomes evident near the transition. With reference to the graph for $q_{\mathrm{i}}=1$ in Fig. 4, we note that the discontinuous volume phase transition occurs somewhere between the swollen state near $\lambda=6 \sigma$ and the collapsed state near $\lambda=7 \sigma$. The annotations of $E^{+}, E^{-}, S^{+}$, and $S^{-}$near these two states on the graph refer qualitatively to the energy and the entropy of the two states. In the swollen state, the unfavorably higher electrostatic energy $\left(E^{+}\right)$is largely compensated for by the greater counterion excluded-volume entropy $\left(S^{+}\right)$; in the collapsed state, the lower electrostatic energy $\left(E^{-}\right)$ balances the loss of counterion excluded-volume entropy $\left(S^{-}\right)$. It is also interesting to note that this energy-entropy interplay involves both long-range electrostatic interactions and short-range excluded-volume interactions.

\section{Structural features}

The discontinous volume phase transitions in polyelectrolyte networks also appear to be evident when we examine certain structural aspects of the network backbone. The single-chain structure factors $S(k)$ for the various polyelectrolyte networks, normalized with respect to the chain length, are shown in Fig. 5. The single-chain structure factors in the swollen states (solid curves) give Flory exponents $\nu$ in the range of $0.8-1$, while in the collapsed states (dashed curves) they give Flory exponents in the range of $0.45-0.5$. Hence the network backbone chains take on nearly Gaussian conformations in collapsed networks but have globally extended conformations in swollen gels. This result is inconsistent with the Gaussian chain ideality assumed in the FloryRehner network. ${ }^{15}$ In Fig. 6 we show the radial distribution function $g_{\mathrm{nn}}(r)$ for crosslink node-crosslink node pairs, where the abscissa has been normalized with respect to the length of the unit cell $L$. The radial distribution functions for swollen gels all show distinctive peaks at $\sqrt{3} L / 4$ $\approx 0.433 L, \sqrt{2} L / 2 \approx 0.707 L$, and $\sqrt{11} L / 4 \approx 0.829 L$, characteristic of the diamond lattice (solid curves), but for collapsed networks the radial distributions almost universally collapse into master curves (dashed curves). The affine network as- 

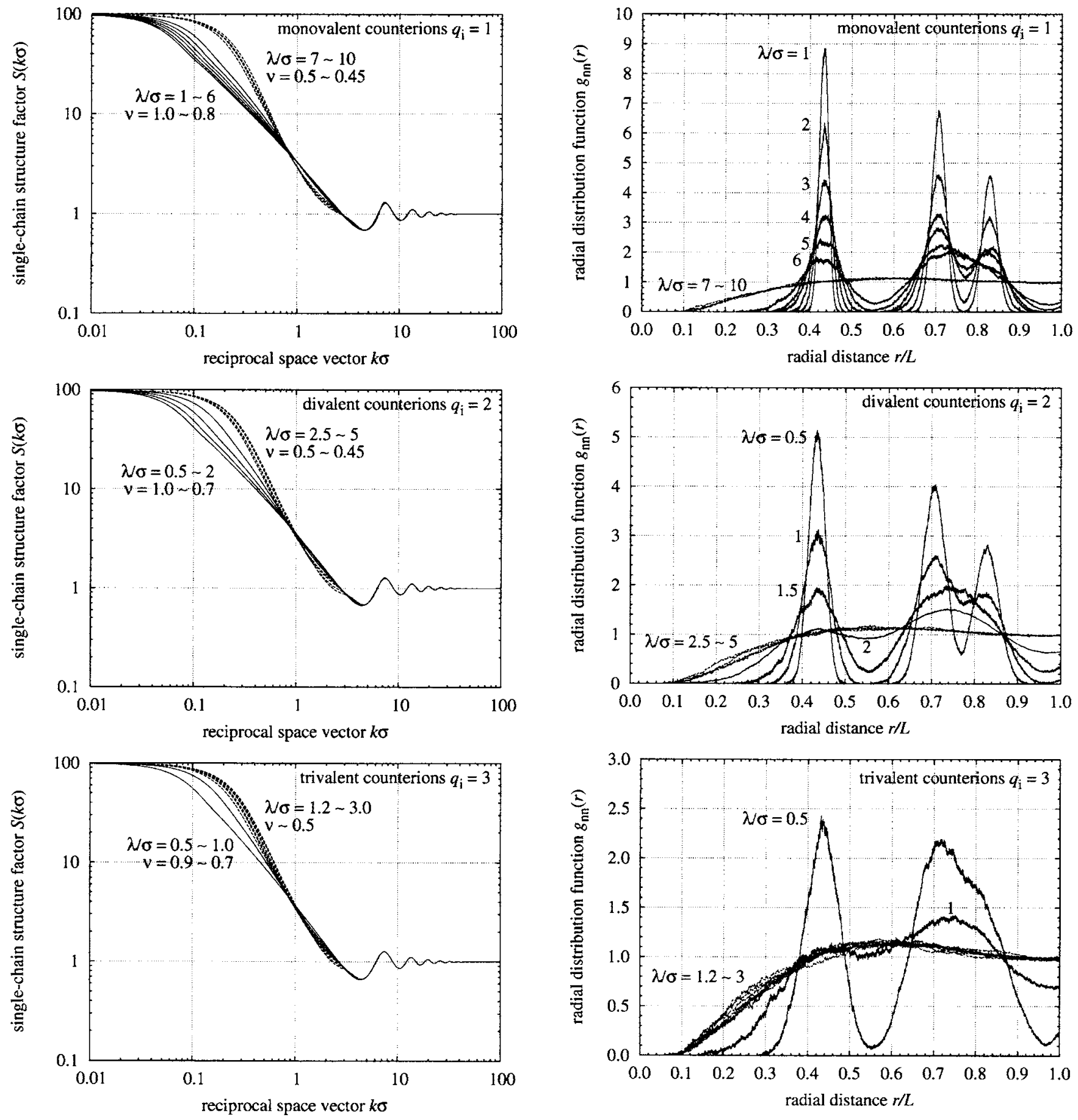

FIG. 5. Single-chain structure factors $S(\boldsymbol{k})$ for polylectrolyte networks with mono-, di-, and trivalent counterions at $T=\varepsilon / k_{\mathrm{B}}$ and $\pi=0$. The solid and dashed curves represent respectively the swollen and collapsed states of the networks. The Bjerrum length $\lambda$ increases and the Flory exponent $\nu$ decreases from left to right.

sumption would therefore seem to hold in swollen polyelectrolyte gels, but clearly not in collapsed polyelectrolyte networks.

\section{SUMMARY}

We performed molecular dynamics simulations of highly-charged defect-free crosslinked polyelectrolyte networks with explicit mono-, di-, or trivalent counterions in good solvent of various dielectricities. In spite of the

FIG. 6. Crosslink node-crosslink node radial distribution function for polyelectrolyte networks with mono-, di-, and trivalent counterions at $T=\varepsilon / k_{\mathrm{B}}$ and $\pi=0$. The solid and dashed curves represent respectively the swollen and collapsed states of the networks. The Bjerrum length $\lambda$ increases from top to bottom.

simplicity of the model, we are able to observe evidence of discontinuous volume phase transitions in polyelectrolyte networks. A detailed examination of the virial terms contributing to the osmotic pressure reveals several important insights into the physical origin of these transitions. The elasticity of polyelectrolyte networks is primarily an entropic effect and is qualitatively consistent with the description of ideal entropic networks. The loss of counterion entropy due to increased strength of the electrostatic interactions and the accompanying network contraction or collapse results in 
increased contributions to both the ideal and excess parts of the osmotic pressure. The overall electrostatic interactions between the charged monomers and counterions are energetic in nature; the resulting cohesion balances the loss of counterion entropy, providing the necessary thermodynamic basis that gives rise to the discontinuous volume phase transitions in polyelectrolyte networks. Finally, an examination of the structure of swollen polyelectrolyte networks shows that the swollen gels are affine networks consisting of wellextended constituent chains, while in the collapsed state the network chains are nearly Gaussian and the topology is not affine.

\section{ACKNOWLEDGMENTS}

We are grateful for the financial support received from the Chemical Sciences, Geosciences, and Biosciences Division, Office of Basic Energy Sciences, Office of Science, U.S. Department of Energy (DE-FG02-99ER14961), the Natural Sciences and Engineering Research Council of Canada (PGSB-242497-2001), and the University of Wisconsin-Madison Grainger Graduate Fellowship Fund. We also wish to acknowledge Professor David A. Hoagland for stimulating discussions concerning this work.

${ }^{1}$ T. Tanaka, in Polyelectrolyte Gels: Properties, Preparation, and Applications, ACS Symposium Series, Vol. 480, edited by R. S. Harland and R. K. Prud'homme (Americal Chemical Society, Washington D.C., 1992), Chap. 1, pp. 1-21.

${ }^{2}$ H. Dautzenberg, W. Jaeger, J. Kötz, B. Philipp, C. Seidel, and D. Stscherbina, Polyelectrolytes: Formation, Characterization and Application (Hanser, Munich, Germany, 1994).

${ }^{3}$ Responsive Gels: Volume Transitions I, Advances in Polymer Science Vol. 109, edited by K. Dušek (Springer-Verlag, Berlin, Germany, 1993).

${ }^{4}$ Responsive Gels: Volume Transitions II, Advances in Polymer Science Vol. 110, edited by K. Dušek (Springer-Verlag, Berlin, Germany, 1993).

${ }^{5}$ C. L. Voycheck and J. S. Tan, in Polyelectrolytes: Science and Technology, edited by M. Hara (Marcel Dekker, New York, 1993), Chap. 6, pp. 299-388.

${ }^{6}$ M. Shibayama and T. Tanaka, in Responsive Gels: Volume Transitions I, Advances in Polymer Science Vol. 109, edited by K. Dušek (SpringerVerlag, Berlin, Germany, 1993).

${ }^{7}$ T. L. Hill, J. Chem. Phys. 20, 1259 (1952).

${ }^{8}$ T. L. Hill, Discuss. Faraday Soc. 13, 132 (1953).

${ }^{9}$ T. L. Hill, An Introduction to Statistical Thermodynamics (AddisonWesley, Reading, MA, 1960), reprinted by Dover Publications, Mineola, New York in 1986.

${ }^{10}$ A. Yu. Zubarev, F. A. Blyakhman, G. H. Pollack, P. Gusev, and A. P. Safronov, Macromol. Theory Simul. 13, 697 (2004).

${ }^{11}$ T. Tanaka, D. Fillmore, S.-T. Sun, I. Nishio, G. Swislow, and A. Shah, Phys. Rev. Lett. 45, 1636 (1980).

${ }^{12}$ T. Tanaka, Phys. Rev. Lett. 40, 820 (1978).

${ }^{13}$ E. Kokufuta, in Physical Chemistry of Polyelectrolytes, Surfactant Science Series, Vol. 99, edited by T. Radeva (Marcel Dekker, New York, 2001), Chap. 17, pp. 591-664.

${ }^{14}$ K. Dušek and D. Patterson, J. Polym. Sci., Part A-2, 6, 1209 (1968).

${ }^{15}$ P. J. Flory, Principles of Polymer Chemistry (Cornell University Press, Ithaca, New York, 1953).

${ }^{16}$ W. W. Graessley, Polymeric Liquids and Networks: Structure and Properties (Garland Science, New York, 2003).

${ }^{17}$ M. Rubinstein and R. H. Colby, Polymer Physics, 1st ed. (Oxford University Press, Oxford, U.K., 2003).

${ }^{18}$ S. Panyukov and Y. Rabin, Phys. Rep. 269, 1 (1996).

${ }^{19}$ R. Everaers and K. Kremer, Phys. Rev. E 53, R37 (1996).

${ }^{20}$ M. Pütz, K. Kremer, and R. Everaers, Phys. Rev. Lett. 84, 298 (2000).

${ }^{21}$ F. A. Escobedo and J. J. de Pablo, J. Chem. Phys. 106, 793 (1997).

${ }^{22}$ F. A. Escobedo and J. J. de Pablo, Mol. Phys. 90, 437 (1997).

${ }^{23}$ F. A. Escobedo and J. J. de Pablo, J. Chem. Phys. 110, 1290 (1999).
${ }^{24}$ F. A. Escobedo and J. J. de Pablo, Phys. Rep. 318, 85 (1999).

${ }^{25}$ G. S. Manning, J. Chem. Phys. 51, 924 (1969).

${ }^{26}$ G. S. Manning, J. Chem. Phys. 51, 934 (1969).

${ }^{27}$ G. S. Manning, J. Chem. Phys. 51, 3249 (1969).

${ }^{28}$ G. S. Manning, Annu. Rev. Phys. Chem. 23, 117 (1972).

${ }^{29}$ G. S. Manning and J. Ray, J. Biomol. Struct. Dyn. 16, 461 (1998).

${ }^{30}$ M. Muthukumar, J. Chem. Phys. 105, 5183 (1996).

${ }^{31}$ M. Muthukumar, J. Chem. Phys. 107, 2619 (1997).

${ }^{32}$ F. Oosawa, Polyelectrolytes (Marcel Dekker, New York, 1971).

${ }^{33}$ S. A. Rice and M. Nagasawa, Polyelectrolyte Solutions: A Theoretical Introduction, Molecular Biology: An International Series of Monographs and Textbooks, Vol. 2 edited by N. O. Kaplan, and H. A. Scheraga (Academic, New York, 1961).

${ }^{34}$ H. H. Hooper, S. Beltran, A. P. Sassi, H. W. Blanch, and J. M. Prausnitz, J. Chem. Phys. 93, 2715 (1990).

${ }^{35}$ H. H. Hooper, H. W. Blanch, and J. M. Prausnitz, Macromolecules 23, 4820 (1990).

${ }^{36}$ M. J. Stevens and K. Kremer, J. Chem. Phys. 103, 1669 (1995).

${ }^{37}$ U. Micka, C. Holm, and K. Kremer, Langmuir 15, 4033 (1999).

${ }^{38}$ M. Deserno, A. Arnold, and C. Holm, Macromolecules 36, 249 (2003).

${ }^{39}$ M. Deserno, C. Holm, and K. Kremer, in Physical Chemistry of Polyelectrolytes, Surfactant Science Series, Vol. 99, edited by T. Radeva (Marcel Dekker, New York, 2001), Chap. 2, pp. 59-110.

${ }^{40}$ H. Schäfer and C. Seidel, Macromolecules 30, 6658 (1997)

${ }^{41}$ Polymeric Systems, Advances in Chemical Physics, Vol. 94 edited by I. Prigogine and S. A. Rice (Wiley, New York, 1996).

${ }^{42}$ S. Panyukov and Y. Rabin, Macromolecules 29, 8530 (1996).

${ }^{43}$ H. Frusawa, K. Ito, and R. Hayakawa, J. Phys. Soc. Jpn. 66, 925 (1997).

${ }^{44}$ H. H. Hooper, J. P. Baker, H. W. Blanch, and J. M. Prausnitz, Macromolecules 23, 1096 (1990).

${ }^{45}$ M. M. Prange, H. H. Hooper, and J. M. Prausnitz, AIChE J. 35, 803 (1989).

${ }^{46}$ M. J. Marchetti, S. Prager, and E. L. Cussler, Macromolecules 23, 1760 (1990).

${ }^{47}$ M. J. Marchetti, S. Prager, and E. L. Cussler, Macromolecules 23, 3445 (1990).

${ }^{48}$ G. Maurer and J. M. Prausnitz, Fluid Phase Equilib. 115, 113 (1996).

${ }^{49}$ Y. Kirokawa and T. Tanaka, J. Chem. Phys. 81, 6370 (1984).

${ }^{50}$ D. P. Aalberts, J. Polym. Sci., Part B: Polym. Phys. 34, 1127 (1996).

${ }^{51}$ Q. Yan and J. J. de Pablo, Phys. Rev. Lett. 91, 018301 (2003).

${ }^{52}$ S. Schneider and P. Linse, Eur. Phys. J. E 8, 457 (2002).

${ }^{53}$ S. Schneider and P. Linse, J. Phys. Chem. B 107, 8030 (2003).

${ }^{54}$ S. Schneider and P. Linse, Macromolecules 37, 3850 (2004).

${ }^{55}$ S. Edgecombe, S. Schneider, and P. Linse, Macromolecules 37, 10089 (2004).

${ }^{56}$ B. A. Mann, R. Everaers, C. Holm, and K. Kremer, Europhys. Lett. 67, 786 (2004).

${ }^{57}$ B. A. Mann, C. Holm, and K. Kremer, J. Chem. Phys. 122, 154903 (2005).

${ }^{58}$ Z.-Y. Lu and R. Hentschke, Phys. Rev. E 67, 061807 (2003).

${ }^{59}$ D. A. McQuarrie, in Statistical Mechanics, Harper's Chemistry Series (Harper \& Row, New York, 1976).

${ }^{60}$ D. Chandler and J. D. Weeks, Phys. Rev. Lett. 25, 149 (1970).

${ }^{61}$ J. D. Weeks, D. Chandler, and H. C. Andersen, J. Chem. Phys. 54, 5237 (1971).

${ }^{62}$ H. C. Andersen, J. D. Weeks, and D. Chandler, Phys. Rev. A 4, 1597 (1971).

${ }^{63}$ R. B. Bird, C. F. Curtiss, R. C. Armstrong, and O. Hassager, Dynamics of Polymeric Liquids: Kinetic Theory, 2nd ed. (Wiley, New York, 1987), Vol. 2.

${ }^{64}$ H. R. Warner, Jr., Ind. Eng. Chem. Fundam. 11, 379 (1972).

${ }^{65}$ S. W. de Leeuw, J. W. Perram, and E. R. Smith, Proc. R. Soc. London, Ser. A 373, 27 (1980).

${ }^{66}$ M. P. Allen and D. J. Tildesley, Computer Simulation of Liquids (Oxford University Press, Oxford, U.K., 1987).

${ }^{67}$ D. Frenkel and B. Smit, Understanding Molecular Simulation: From Algorithms to Applications, Computational Science Series Vol. 1, 2nd ed. (Academic, San Diego, California, 1996).

${ }^{68}$ D. Fincham, Mol. Simul. 13, 1 (1994).

${ }^{69}$ G. J. Martyna, M. L. Klein, and M. E. Tuckerman, J. Chem. Phys. 97, 2635 (1992).

${ }^{70}$ G. J. Martyna, M. E. Tuckerman, D. J. Tobias, and M. L. Klein, Mol. Phys. 87, 1117 (1996). 